\title{
Valor económico de la fibra de vicuña en la Reserva Nacional Salinas y Aguada Blanca (Arequipa, Perú)
}

\author{
Economic value of the vicuña fiber in Salinas y Aguada Blanca National Reserve \\ (Arequipa, Perú)
}

\author{
César Edgardo Medina ${ }^{1,2 *}$, Yasmy Karina Medina ${ }^{1,2}$ y Edwin Fredy Bocardo ${ }^{2}$
}

\begin{abstract}
RESUMEN
La valoración económica de la diversidad biológica ha tenido un gran avance conceptual y metodológico en los años 90 . Sin embargo, muy poco se ha logrado en su puesta en marcha para aplicaciones prácticas y generación de políticas públicas en Arequipa, Perú. Por tanto, el presente estudio valoró económicamente la cantidad de fibra de vicuña que se puede obtener de la población de vicuñas que habitan en la Reserva Nacional Salinas y Aguada Blanca (RNSAB). Para ello, se realizaron censos en transectos lineales de ancho variable y se calculó la densidad poblacional de vicuñas. Luego se recopiló información de la Gerencia Regional de Agricultura de Arequipa (GRAG) y se estimó porcentaje promedio de vicuñas esquiladas, peso promedio de fibra que se puede obtener en un animal y precio promedio de un kilo de fibra. Como resultado, se estimó una densidad poblacional de vicuñas de 0.038457 individuos/ha para la RNSAB, y al ser extrapolada a la superficie de la Reserva y la información de la GRAG se obtuvo un valor económico de U\$D 384.209,60 por concepto de aprovechamiento de fibra de vicuña. Dicho valor se convierte en un referente para la contabilidad nacional y la generación de instrumentos de gestión ambiental para un aprovechamiento sostenible del recurso natural.
\end{abstract}

Palabras clave: Vicugna vicugna, densidad poblacional, valoración económica

\begin{abstract}
The economic valuation of biological diversity has had a conceptual and methodological breakthrough in the 90s, however, very little has been achieved in its implementation for practical applications and generation of public policies in Arequipa, Peru. Therefore, this study economically assessed the amount of vicuña fiber that can be obtained from the population of vicuñas that inhabit in the Salinas y Aguada Blanca National Reserve (RNSAB). First of all, census was carried out on linear transects of variable width and the population density of vicuñas was calculated. Secondly, information from the Regional Management of Agriculture of Arequipa (GRAG) was collected and it was estimated: average percentage of sheared vicuñas, average weight of fiber that can be obtained in an animal, and average cost of one kilo of fiber. As a result, the population density of vicuñas was 0.038457 individuals/ha in the RNSAB, amount that if was extrapolated to the surface of the Reserve and the information of the GRAG, an economic value of US \$ 384,209.60 is obtained by the exploitation of vicuñ fiber. This value becomes a benchmark for national accounting and the generation of environmental management instruments for a sustainable use of the natural resource.
\end{abstract}

Palabras clave: Vicugna vicugna, populational density, economic valuation

\section{Introducción}

Los camélidos sudamericanos comprenden cuatro especies: la llama (Lama glama), la alpaca (Lama pacos), el guanaco (Lama guanicoe) y la vicuña (Vicugna vicugna). La llama y la alpaca son especies domésticas, mientras que el guanaco y la vicuña son especies silvestres. El Perú es el principal productor

1 Universidad Nacional de San Agustín de Arequipa, Museo de Historia Natural - Colección Científica (MUSA). Arequipa, Perú.

2 Universidad Nacional de San Agustín de Arequipa, Departamento Académico de Biología, Sección Zoología. Arequipa, Perú.

* Autor por correspondencia: cmedinap@unsa.edu.pe; cmedinap1234@yahoo.com

Fecha de Recepción: 22 agosto, 2018.

Fecha de Aceptación: 13 septiembre, 2018.

DOI: 
de camélidos sudamericanos del mundo con poco más de 5 millones de cabezas entre las cuatro especies, de las cuales, 3 millones 596 mil 753 son alpacas y representan más del $85 \%$ de las existentes en el mundo (CONACS, 2005).

La vicuña presenta una de las fibras de origen animal más finas del mundo, la cual se utiliza como insumo para la confección de telas y prendas de vestir de alta calidad, con una demanda concentrada principalmente en Italia, Inglaterra, Alemania y Estados Unidos (FIA, 2008). La población de vicuñas se estima en 302.210 ejemplares, y los principales países productores de fibra son Perú, Argentina y Bolivia (CONACS, 2005). En Perú, la estrategia utilizada para el desarrollo del mercado para la fibra de vicuña se basa en la integración de políticas del Estado, la empresa privada y empresas textiles. Esto origina que exista una oferta permanente de fibra en el mercado, con precios comunales medios y un alto valor agregado (Galaz y Gonzales, 2005).

En el año 1979 fue creada la Reserva Nacional de Salinas y Aguada Blanca(RNSAB), con el propósito de conservar y manejar las poblaciones de vicuñas para el aprovechamiento de su fibra (Zeballos et al., 2010; Davies, 2003). Para ello, se han desarrollado dos modalidades de manejo de vicuñas: manejo en cautiverio y manejo en silvestría, y esta última es la que presenta mayores dificultades para obtener cálculos de aprovechamiento para toda la RNSAB.

Si bien la valoración económica de bienes y servicios que brinda la diversidad biológica de las Áreas Naturales Protegidas (ANP) tuvo un gran avance conceptual y metodológico en los años 90 (Glave y Pizarro, 2001), muy poco se ha logrado en términos de su puesta en marcha en aplicaciones prácticas y políticas públicas en el sur del Perú. Por tanto, el presente estudio tuvo como objetivo valorar económicamente la producción de fibra de vicuña en la RNSAB, basado en los lineamientos propuestos por la legislación nacional (MINAM, 2014; MINAM, 2015).

\section{Metodología}

\section{Área de estudio}

La Reserva Nacional de Salinas y Aguada Blanca (RNSAB) es una de las principales ANP del Perú, que resguarda una muestra representativa de la Puna Seca en el suroeste del país (Figura 1). Se ubica en los departamentos de Arequipa y Moquegua, y ocupa un área de 366.936 ha, en un rango altitudinal que va de los 2.800 a más de $6.000 \mathrm{~m}$ de altitud.

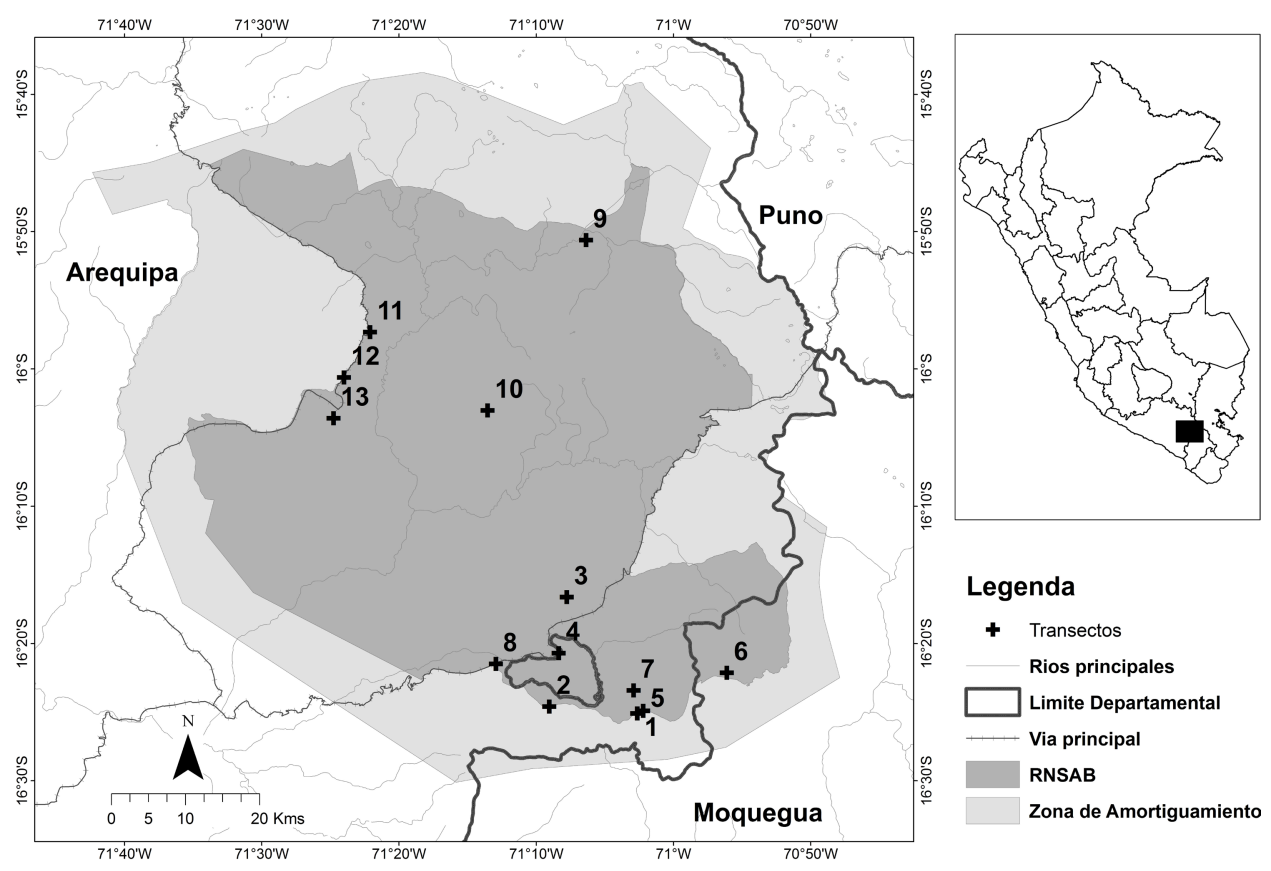

Figura 1. Mapa de ubicación del área de estudio, mostrando los puntos iniciales de los transectos evaluados en la Reserva Nacional Salinas y Aguada Blanca. 
La flora de la RNSAB consta de más de 463 especies de plantas vasculares, y las Asteraceae y Poaceae son las familias con mayor número de especies; mientras que la fauna de vertebrados suma 207 especies, conformadas por 37 mamíferos (34 nativos y 3 introducidos en estado silvestre), 158 aves, 5 reptiles, 4 anfibios y 3 peces ( 2 nativos y 1 introducido) (Zeballos et al., 2010).

\section{Métodos}

La densidad poblacional de vicuñas en la RNSAB fue evaluada con ayuda de censos en transectos lineales (Tellería, 1986), durante el mes de febrero de 2018 (Tabla 1). Para ello, se utilizaron las principales vías de acceso que existen en la RNSAB, las cuales fueron recorridas en camioneta a una velocidad promedio de $20 \mathrm{~km}$ por hora, entre las 8:00 y las 11:00 hrs. Los avistamientos fueron realizados por dos personas, y se registró el número de individuos y la distancia perpendicular de los individuos al transecto, ubicados dentro del ANP (Soriguer et al., 1997). La densidad de vicuñas fue calculada con ayuda de los estimadores implementados en el Programa Distance 6.0 (Buckland et al., 2004).

La Gerencia Regional de Agricultura de Arequipa (GRAG) es la entidad del Estado encargada de promover y fiscalizar el aprovechamiento de la fibra de vicuña, motivo por el cual se solicitó la siguiente información a la GRAG: a) número de vicuñas esquiladas; b) peso de fibra de vicuña que se obtiene de cada animal esquilado; c) precio del kilo de fibra

Tabla 1. Ubicación de los transectos evaluados y esfuerzo de muestreo empleado para estimar la densidad poblacional de vicuñas en la Reserva Nacional Salinas y Aguada Blanca.

\begin{tabular}{|c|c|c|c|c|c|c|c|c|}
\hline \multirow{2}{*}{$\begin{array}{c}\text { ID } \\
\text { Transecto }\end{array}$} & \multirow[b]{2}{*}{ Localidad de Referencia } & \multirow{2}{*}{$\begin{array}{l}\text { Longitud del } \\
\text { Transecto } \\
\text { (km) }\end{array}$} & \multicolumn{4}{|c|}{ Coordenadas geográficas } & \multirow[b]{2}{*}{ Réplicas } & \multirow{2}{*}{$\begin{array}{c}\text { Kilómetros } \\
\text { censados }\end{array}$} \\
\hline & & & Punto & $S$ & $\mathrm{O}$ & $\begin{array}{l}\text { Altitud } \\
\text { (m) }\end{array}$ & & \\
\hline \multirow{2}{*}{1} & \multirow{2}{*}{ Santa Lucia de Salinas } & \multirow{2}{*}{13,04} & Inicio & $16^{\circ} 25^{\prime} 6,21^{\prime \prime}$ & $71^{\circ} 22^{\prime} 38,09 ”$ & 4361 & \multirow{2}{*}{4} & \multirow{2}{*}{52,16} \\
\hline & & & Final & $16^{\circ} 24^{\prime} 36,08^{\prime \prime}$ & $71^{\circ} 9^{\prime} \quad 2,61^{\prime \prime}$ & 4368 & & \\
\hline \multirow{2}{*}{2} & \multirow{2}{*}{ Salinas Moche } & \multirow{2}{*}{3,70} & Inicio & $16^{\circ} 24^{\prime} 36,08^{\prime \prime}$ & $71^{\circ} 9^{\prime} 2,61^{\prime \prime}$ & 4368 & \multirow{2}{*}{14} & \multirow{2}{*}{51,80} \\
\hline & & & Final & $16^{\circ} 23^{\prime} 29,39^{\prime \prime}$ & $71^{\circ} 10^{\prime} 39,56^{\prime \prime}$ & 4331 & & \\
\hline \multirow{2}{*}{3} & \multirow{2}{*}{ Salinas Huito 1} & \multirow{2}{*}{7,53} & Inicio & $16^{\circ} 16^{\prime} 37,57^{\prime \prime}$ & $71^{\circ} 7^{\prime} 46,56^{\prime \prime}$ & 4474 & \multirow{2}{*}{3} & \multirow{2}{*}{22,59} \\
\hline & & & Final & $16^{\circ} 14^{\prime} 30,48^{\prime \prime}$ & $71^{\circ} 10^{\prime} 27,50^{\prime \prime}$ & 4753 & & \\
\hline \multirow{2}{*}{4} & \multirow{2}{*}{ Salinas Huito 2} & \multirow{2}{*}{9,05} & Inicio & $16^{\circ} 20^{\prime} 42,22^{\prime \prime}$ & $71^{\circ} 8^{\prime} 21,88^{\prime \prime}$ & 4326 & \multirow{2}{*}{1} & \multirow{2}{*}{9,05} \\
\hline & & & Final & $16^{\circ} 21^{\prime} 29,54^{\prime \prime}$ & $71^{\circ} 12^{\prime} 56,38^{\prime \prime}$ & 4303 & & \\
\hline \multirow{2}{*}{5} & \multirow{2}{*}{ Vía Ubinas } & \multirow{2}{*}{16,09} & Inicio & $16^{\circ} 24^{\prime} 54,75^{\prime \prime}$ & $71^{\circ} 2^{\prime} 12,43^{\prime \prime}$ & 4366 & \multirow{2}{*}{2} & \\
\hline & & & Final & $16^{\circ} 22^{\prime} 37,36^{\prime \prime}$ & $70^{\circ} 55^{\prime} 27,28^{\prime \prime}$ & 4402 & & 32,11 \\
\hline 6 & & 870 & Inicio & $16^{\circ} 22^{\prime} 8,44^{\prime \prime}$ & $70^{\circ} 56^{\prime} \quad 7,05^{\prime \prime}$ & 4456 & 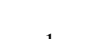 & 070 \\
\hline 0 & via Canteosanti & 0,72 & Final & $16^{\circ} 17^{\prime} 51,24^{\prime \prime}$ & $70^{\circ} 55^{\prime} 37,95^{\prime \prime}$ & 4489 & 1 & 0,72 \\
\hline & & & Inicio & $16^{\circ} 23^{\prime} 25,12^{\prime \prime}$ & $71^{\circ} 2^{\prime} 54,49^{\prime \prime}$ & 4339 & & \\
\hline 1 & тигараmpa & 17,10 & Final & $16^{\circ} 17^{\prime} 27,46^{\prime \prime}$ & $70^{\circ} 56^{\prime} 31,30^{\prime \prime}$ & 4433 & 1 & $17, / 0$ \\
\hline & & & Inicio & $16^{\circ} 21^{\prime} 29,54^{\prime \prime}$ & $71^{\circ} 12^{\prime} 56,38^{\prime \prime}$ & 4304 & & \\
\hline 0 & L1 SImUlal & $10,4 J$ & Final & $16^{\circ} 22^{\prime} 23,87^{\prime \prime}$ & $71^{\circ} 18^{\prime} 10,13^{\prime \prime}$ & 4272 & 2 & 20,90 \\
\hline D & 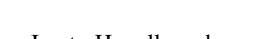 & A 4 & Inicio & $15^{\circ} 50^{\prime} 36,91^{\prime \prime}$ & $71^{\circ}$ 6'22,03'” & 4454 & 年 & 10776 \\
\hline 3 & 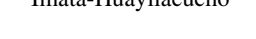 & 34,44 & Final & $16^{\circ} 1^{\prime} 41,83^{\prime \prime}$ & $71^{\circ} 16^{\prime} 53,70^{\prime \prime}$ & 4245 & 4 & $15 /, 70$ \\
\hline 10 & He 11 b $\mathrm{C}$. & 90 & Inicio & $16^{\circ} 3^{\prime} 1,58^{\prime \prime}$ & $71^{\circ} 13^{\prime} 32,71^{\prime \prime}$ & 4455 & 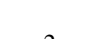 & PO \\
\hline 10 & Huayllacucho-Condorı & 14,30 & Final & $16^{\circ} 1^{\prime} 42,09$ ' & $71^{\circ} 7 ' 59,95^{\prime \prime}$ & 4372 & 2 & 28,60 \\
\hline 10 & Sh & 000 & Inicio & $15^{\circ} 57^{\prime} 19,44^{\prime \prime}$ & $71^{\circ} 22^{\prime} \quad 6,59^{\prime \prime}$ & 4274 & 0 & 4101 \\
\hline 11 & Sumbay-Alcanuito & 20,90 & Final & $15^{\circ} 47^{\prime} 25,00^{\prime \prime}$ & $71^{\circ} 22^{\prime} 48,45^{\prime \prime}$ & 4317 & 2 & 41,81 \\
\hline 12 & S & 3040 & Inicio & $16^{\circ} 0 ’ 37,46^{\prime \prime}$ & $71^{\circ} 24^{\prime} 0,75^{\prime \prime}$ & 4080 & 6 & 0 \\
\hline 12 & sumbay-1ocra & 28,48 & Final & $15^{\circ} 50^{\prime} 5,31^{\prime \prime}$ & $71^{\circ} 28^{\prime} 12,93^{\prime \prime}$ & 4359 & 0 & $1 / 0,88$ \\
\hline 12 & 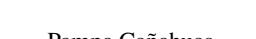 & 1015 & Inicio & $16^{\circ} 3 ’ 35,59^{\prime \prime}$ & $71^{\circ} 24^{\prime} 46,37^{\prime \prime}$ & 4022 & 1 & 1015 \\
\hline 15 & rampa Cananuas & $10,1 J$ & Final & $16^{\circ} 12^{\prime} 45,45^{\prime \prime}$ & $71^{\circ} 26^{\prime} 11,56^{\prime \prime}$ & 4195 & 1 & $10,1 J$ \\
\hline & & & & & Esfuerz & muestreo & 43 & 612,28 \\
\hline
\end{tabular}


de vicuña en los últimos 10 años. Dicha información fue resumida en porcentajes y promedios, los cuales se extrapolaron a la población estimada de vicuñas que habitan en la RNSAB, para obtener finalmente el valor económico asociado al aprovechamiento de la fibra de vicuña en el ámbito del ANP.

\section{Densidad}

\section{Resultados}

Durante los censos en transectos lineales se obtuvo un total de 202 avistamientos de vicuña, utilizando un esfuerzo de muestreo total de $612.28 \mathrm{~km}$ censados distribuidos en 43 transectos recorridos (Tabla 1).

Según los análisis del Programa Distance 6,2, la función Hazard Rate, con la serie de expansión Hermite polinomial, fue el modelo que mejor se ajustó a la distribución de las distancias perpendiculares registradas $(\mathrm{AIC}=2482,2)$, estimando una probabilidad de detección de 20,13\% (IC 95\% = 15.988 - 25.347; CV = 11,72\%), una amplitud de banda efectiva de 181,17 $\mathrm{m}$ (IC 95\% = 143,89 - 228,12; $\mathrm{CV}=11,72 \%$ ) y un tamaño promedio de grupos familiares de vicuña de 4,22 individuos/grupo (IC $95 \%=3.7562-4,7495 ; \mathrm{CV}=5,95 \%$ ) (Figura 2).

El modelo estimó una densidad poblacional de 0,0091049 grupos de vicuña/hectárea (IC 95\% = $0,0057142-0,014508 ; \mathrm{CV}=23,69 \%$ ) y de 0,038457 individuos/hectárea (IC 95\% =0,023820 - 0,062088; $\mathrm{CV}=24,43 \%)$. El componente que más aportó a la varianza de la densidad fue la tasa de encuentro $71 \%$. Dichos valores de densidad presentaron bajos valores de variación $(\mathrm{CV}<30 \%)$, corroborando la confiabilidad de cálculos realizados.
Considerando la densidad de vicuñas obtenida durante el presente estudio y la superficie de la RNSAB (366.936 ha), se estimó una población de 14.111 vicuñas que habitan en la RNSAB.

\section{Valoración económica}

La información proporcionada por la GRAG mostró que la venta de fibra de vicuña no responde a un modelo económico básico de libre oferta y demanda, debido a que el precio por kilogramo de fibra se mantuvo relativamente estable durante seis años de comercialización (U\$D 352,86). Además, se observó que, en promedio, sólo el 39,98\% de las vicuñas capturadas fueron esquiladas (Tabla 2).

Considerando una población de 14.111 vicuñas para la RNSAB, el porcentaje promedio de vicuñas esquiladas, el peso promedio de fibra que se puede obtener en un animal y el precio promedio de un kilo de fibra, el valor económico generado por el aprovechamiento de fibra de vicuña en la RNSAB alcanzó un total de U\$D 384.209,60.

\section{Discusiones}

La valoración económica de servicios ambientales es un tema relativamente nuevo en el Perú, para el cual existen limitados estudios en ANP (Glave y Pizarro, 2001; Loyola, 2007; Gómez et al., 2012). Uno de los primeros esfuerzos por conocer el valor económico de la diversidad biológica en ANP del Perú fue desarrollado por Glave y Pizarro (2001), quienes, a través de un programa de becas en investigación, capacitaron a profesionales del sector

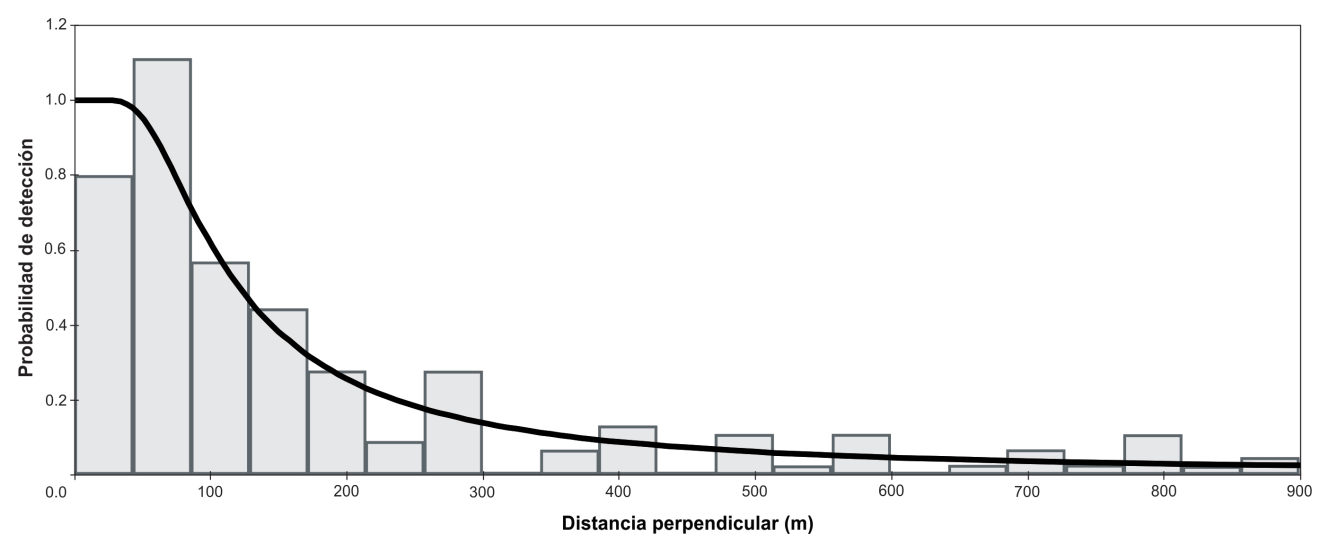

Figura 2. Probabilidad de detección en los transectos de evaluación para vicuñas en la Reserva Nacional Salinas y Aguada Blanca. 
Tabla 2. Estadísticas del manejo de vicuña en el ámbito de la Reserva Nacional Salinas y Aguada Blanca, durante los últimos 10 años (GRAG, 2018). El asterisco señala los datos considerados para la estimación de valores promedio.

\begin{tabular}{|c|c|c|c|c|c|c|}
\hline Año & $\begin{array}{l}\mathrm{N}^{\circ} \text { de vicuñas } \\
\text { capturadas }\end{array}$ & $\begin{array}{l}\mathrm{N}^{\circ} \text { de vicuñas } \\
\text { esquiladas }\end{array}$ & $\begin{array}{l}\text { \% de vicuñas } \\
\text { esquiladas }\end{array}$ & $\begin{array}{l}\text { Producción fibra } \\
\text { total (kg.) }\end{array}$ & $\begin{array}{l}\text { Producción } \\
\text { de fibra, por } \\
\text { individuo (gr/ } \\
\text { vicuña) }\end{array}$ & $\begin{array}{l}\text { Precio de fibra de vicuña, sucia } \\
\text { (USD } / \mathrm{kg})\end{array}$ \\
\hline $2007 *$ & 2013 & 894 & 44,41 & 174,45 & 195 & 340 \\
\hline 2008* & 2375 & 1013 & 42,65 & 193,23 & 190 & 370 \\
\hline $2009 *$ & 2857 & 1183 & 41,41 & 241,54 & 204 & 345 \\
\hline 2010 & 3253 & 1470 & 45,19 & 288,34 & 196 & 660 (Descerdada) \\
\hline $2011^{*}$ & 2642 & 972 & 36,79 & 182,40 & 187 & 315 \\
\hline $2012^{*}$ & 2431 & 901 & 37,06 & 164,95 & 183 & 380 \\
\hline $2013^{*}$ & 2513 & 872 & 34,70 & 166,73 & 191 & 360 \\
\hline $2014 *$ & 3574 & 1530 & 42,81 & 308,13 & 201 & 360 \\
\hline 2015 & 4587 & 2471 & 53,87 & 505,15 & 204 & 400 (clasificada) \\
\hline 2016 & 4915 & 1957 & 39,82 & 380,50 & 194 & No comercializada \\
\hline 2017 & 5570 & 2228 & 40,00 & 423,28 & 190 & No comercializada \\
\hline \multicolumn{3}{|c|}{ Promedios } & 39,98 & 204,49 & 193 & 352,86 \\
\hline
\end{tabular}

público ligados al sector ambiental en herramientas de valoración económica.

Dentro de la clasificación de las ANP, las Reservas Nacionales se encuentran destinadas a la conservación de la diversidad biológica y la utilización sostenible, incluso comercial, de los recursos de flora y fauna silvestre bajo planes de manejo (SERNANP, 2018). Como ejemplo, las Reservas Nacionales de Pucacuro y Pacaya Samiria, ubicadas en las regiones de Loreto y Ucayali, han llevado al límite el aprovechamiento de fauna silvestre tras promover la comercialización de carne de monte de "huangana" Tayassu pecari, "sajino" Pecari tajacu, "venado rojo" Mazama americana, "venado gris" Mazama nemorivaga y "majas" Cuniculus paca, con base en estudios previos de valoración económica y bajo estrictos planes de manejo (Aquino et al., 2007; Pérez-Peña et al., 2016).

La RNSAB fue concebida como una nueva área para la conservación y el manejo de la vicuña, en vista de que en la zona se encontraron condiciones óptimas para su supervivencia y se contaba con la experiencia del exitoso programa de manejo de vicuñas de Pampa Galeras (Zeballos et al., 2010). Para el año 1980, la población de vicuñas en la RNSAB se estimaba en 1.367 individuos. Sin embargo, dicho número se ha visto incrementado hasta alcanzar entre los 11 y 12 mil ejemplares para el año 2016 (Walter Vega, com. pers.). Dichos valores fueron obtenidos mediante técnicas de censo tradicionales y utilizando una gran cantidad de personal y recursos logísticos. No obstante, concordamos con Gallardo et al. (2010), quienes, utilizando menor cantidad de recursos y herramientas informáticas, lograron resultados similares a los obtenidos con técnicas de censo tradicionales (Buckland et al., 2004; Pérez-Peña et al., 2016).

Las campañas de esquila de vicuña en la RNSAB se realizan frecuentemente entre los meses de agosto y noviembre de cada año, efectuando la esquila efectiva de sólo el 39,98\% de vicuñas capturadas (GRAG, 2018). En los últimos 10 años, la producción de fibra de vicuña en la RNSAB ha sufrido fluctuaciones importantes, llegando a obtenerse desde $165 \mathrm{~kg}$ de fibra en el año 2012 hasta $505 \mathrm{~kg}$ en 2015, la cual ha sido vendida a un precio relativamente constante durante dicho periodo (U\$D 352 en promedio). Este panorama evidenciaría un monopolio del mercado de la fibra de vicuña, lo cual es preocupante debido a que el Perú es el país con la mayor población de vicuñas de Sudamérica (Quispe et al., 2009).

\section{Conclusiones}

La implementación de un diseño de muestreo adecuado y el uso de herramientas informáticas se presentan como una alternativa rápida y eficiente para estimar la densidad poblacional de vicuñas en la RNSAB.

El valor económico de la fibra de vicuña en la RNSAB fue de 384.209,6 dólares americanos, cifra que se aproxima al monto máximo que se podría obtener por la esquila de toda la población de vicuñas que habitan en la RNSAB. 


\section{Agradecimientos}

Se agradece a la Universidad Nacional de San Agustín de Arequipa (UNSA) por el financiamiento (Contrato de Subvención No TM-0005-2016), habiendo sido ejecutado con el apoyo en la gestión de CIENCIACTIVA y CONCYTEC. También, se agradece al Servicio Nacional de Áreas Naturales Protegidas (SERNANP) por facilitarme la autorización de investigación dentro de la Reserva Nacional de Salinas y Aguada Blanca (RNSAB).

\section{Literatura citada}

Aquino, R.; Pacheco, T.; Vásquez, M.

2007. Evaluación y valorización económica de la fauna silvestre en el río Algodón, Amazonía peruana. Revista Peruana de Biología, 14 (2): 187-192.

Buckland, S.T.; Anderson, D.R.; Burnham; K.P.; Laake, J.L.; Borchers, D.L.; Thomas, L.

2004. Advanced Distance Sampling. Oxford University Press, Oxford. $416 \mathrm{p}$

CONACS.

2005. Informe anual. Consejo Nacional de Camélidos Sudamericanos, Ministerio de Agricultura. Lima. Disponible en: http://www.minagri.gob.pe/portal/?limitstart=0. Consultado: 21/jul/2018.

FIA.

2008. Resultados y lecciones en Producción y Comercialización de fibra de vicuñas, proyectos de innovación en la Región de Arica y Parinacota, y Región de Tarapacá. Fundación para la Innovación Agraria (FIA), Ministerio de Agricultura, Chile. $50 \mathrm{p}$.

Galaz, J.; Gonzales, G.

2005. Técnicas de manejo productivo de la vicuña (Vicugna vicugna Molina 1782) en Chile. Corporación Nacional Forestal, Fundación para la Innovación Agraria (CONAFFIA). $280 \mathrm{p}$.

Gallardo, G.; Núñez, A.; Pacheco, L.F.

2010. Transectos lineales como opción para estimar abundancia de vicuñas (Vicugna vicugna): Estudio de caso en el Parque Nacional Sajama, Bolivia. Ecología en Bolivia, 45 (1): 64-72.

Glave, M.; Pizarro, R.

2001. Valoración Económica de la Diversidad Biológica y Servicios Ambientales en el Perú. Proyecto BIOFOR. INRENA/IRG/USAID. 480 p.

Gómez, R.; Julien, J.; Kámiche, J.

2012. Valoración económica de El Parque Nacional del Río Abiseo: el aporte de los servicios de regulación y soporte. Consorcio de Investigación Económica y Social - Universidad del Pacífico. 103 p.

GRAG.

2018. Cuadro estadístico del manejo de vicuña en el ámbito de la Reserva Nacional Salinas y Aguada Blanca, durante los últimos 10 años. Subgerencia de Promoción Agraria, Gerencia Regional de Agricultura del Gobierno Regional de Arequipa. Informe $N^{\circ}$ 003-2018/DAC.
Loyola, R.

2007. Valoración del Servicio Ambiental de Provisión de Agua con Base en la Reserva Nacional Salinas y Aguada Blanca Cuenca del Río Chili. PROFONANPE. Lima, Perú. 228 p. MINAM.

2014. Guía nacional de valoración económica del patrimonio natural (Resolución Ministerial N ${ }^{\circ} 409-2014$ MINAM). Dirección General de Evaluación, Valoración y Financiamiento del Patrimonio Natural, Ministerio del Ambiente. Lima, Perú. 44 p.

MINAM.

2015. Manual de valoración económica del patrimonio natural. Dirección General de Evaluación, Valoración y Financiamiento del Patrimonio Natural, Ministerio del Ambiente. Lima, Perú. 45 p.

Pérez-Peña, P.; Gonzales-Tanchiva, C.; Trigoso-Pinedo, M. 2016. Evaluación del plan de manejo de animales de caza en la Reserva Nacional Pucacuro. Folia Amazónica, 25 (1): 1-16. Quispe, E.C.; Rodríguez, T.C.; Iñiguez, L.R.; Mueller, J.P. 2009. Producción de fibra de alpaca, llama, vicuña y guanaco en Sudamérica. Animal Genetic Resources Information, 45: 1-14.

Sánchez, J.; Zeballos, H.

2010. La vicuña (Vicugna vicugna mensalis), su conservación y manejo en la Reserva Nacional de Salinas y Aguada Blanca, sur del Perú. En: H. Zeballos, J. Ochoa \& E. López (eds.), Diversidad Biológica de la Reserva Nacional de Salinas y Aguada Blanca. DESCO, INRENA, PROFONANPE. Pp: 49-54.

SERNANP.

2018. ¿Qué es un ANP? Servicio Nacional de Áreas Naturales Protegidas. Lima, Perú. Disponible en: http://www.sernanp. gob.pe. Consultado: 27/jul/2018.

Soriguer, R.C.; Pérez, J.M.; Fandos, P. 1997. Métodos de Mastozoología. Teoría de Censos: Aplicación al caso de los mamíferos. Galemys, 9: 15-37.

Tellería, J.

1986. Manual para el Censo de los Vertebrados Terrestres. Editorial Raíces. Universidad Complutense. Madrid, España. $278 \mathrm{p}$.

Zeballos, H.; Ochoa, J.; López, E.

2010. Diversidad Biológica de la Reserva Nacional de Salinas y Aguada Blanca. DESCO, INRENA, PROFONANPE. Arequipa, Perú. 313 p. 